Article

\title{
Hermite-Hadamard Type Inequalities for the Class of Convex Functions on Time Scale
}

\author{
Saima Rashid 1,2®, Muhammad Aslam Noor ${ }^{2}$, Khalida Inayat Noor ${ }^{2}$, Farhat Safdar ${ }^{3}$ and \\ Yu-Ming Chu $4, *$ (D) \\ 1 Department of Mathematics, Government College University, Faisalabad 38000, Pakistan; \\ saimarashid@gcuf.edu.pk \\ 2 Department of Mathematics, COMSATS University Islamabad, Islamabad 45550, Pakistan; \\ nooraslam@gmail.com (M.A.N.); Khalida@gmail.com (K.I.N.) \\ 3 Department of Mathematics, SBK University, Quetta 87300, Pakistan; Farhat_900@yahoo.com \\ 4 Department of Mathematics, Huzhou University, Huzhou 313000, China \\ * Correspondence: chuyuming2005@126.com
}

Received: 8 September 2019; Accepted: 8 October 2019; Published: 12 October 2019

\begin{abstract}
We investigate a time scale version of two auxiliary functions for the class of convex functions. We derive several novel dynamic inequalities for these classes of convex functions. Applications of these consequences are taken into consideration in special means. Furthermore, illustrative examples are introduced to help our outcomes. Meanwhile, we communicate a few particular cases which may be deduced from our main outcomes.
\end{abstract}

Keywords: convex functions; Hermite-Hadamard inequality; $\left(\Psi_{1}, \Psi_{2}\right)$-convex functions; Time scale

\section{Introduction}

Modeling some global issues requires utilizing a powerful framework that incorporates both discrete and continuous times. This is normal to look at whether it is possible to present a structure that grants us to include both dynamical systems concurrently so that you can probably acquire some perception and adequate knowledge of the elusive variations between discrete and continuous domains. To answer this, an approach was designed by Hilger [1]. The precept goal of dynamic theory on time scales is that it constructs channels between continuous and discrete cases. Afterward, this concept has been built by many researchers, see References [2,3]. It is as dated as classical calculus but it acquires more importance in recent decades, this is due to its applications in various fields such as physics, biology, fluid dynamics, control theory, image processing, signal processing and computer networking. Researchers connected with pure mathematics have implemented time scales calculus in mathematical inequalities to unify discrete and continuous versions of inequalities. In recent years, research has proceeded to unify and amplify integral inequalities through innovative ideas and approaches of time scales $[4,5]$. These integral inequalities are utilized in numerous areas for the boundedness, uniqueness, and so forth, of the solutions to integrodifferential equations $[6,7]$.

Integral inequalities on time scale have also been a topic of debate for a long while. Due to their potentials to be expanded, several variants have been established by many authors, see References [4,5,8-13]. Bohner et al. [6] and Donchev et al. [7] figured various dynamic inequalities that essentially depend on integral inequalities. These sorts of variants are known as Hermite-Hadamard, Ostrowski, Grüss, Hardy and weighted-Ostrowski type variants in the literature. Moreover, researchers $[4,5]$ clarified numerous variants on time scales, which bound together and expanded variants illustrated by Bohner. To demonstrate the theoretical outcomes, it has been validated that the received variants can be utilized as significant apparatuses in the investigation of specific features of dynamic systems on time scales. 
Tahir et al. [11] proposed some variants of Hermite-Hadamard type, consolidating two integral terms, which are themselves the speculations and modifications of several present outcomes on an arbitrary time scale. These acquired outcomes assumed a crucial job in analyzing some classes of integral and integrodifferential equations. In addition, this methodology has fertile utilities in establishing the explicit estimates of certain classes of dynamical frameworks on time scales.

The convexity principle is a powerful and effective approach for studying a massive magnificence of problems that arises in diverse fields of pure and applied sciences. Many new forms had been introduced and investigated of convex sets and convex functions. Several researchers have been derived new variants associated with convex functions, see, for example, References [4,5,11,14-23] and the references therein. Moreover, it is splendid that convexity offers to proliferate ideas and fertile applications in every branch of pure and applied mathematics. Hermite-Hadamard [24,25] proved an inequality involving integral of function and it is known that $\eta: \mathrm{Y}=\left[\varsigma_{1}, \varsigma_{2}\right]_{\mathcal{T}} \rightarrow \mathcal{R}$ is a convex function, if and only if it satisfies the inequality

$$
\eta\left(\frac{\varsigma_{1}+\varsigma_{2}}{2}\right) \leq \frac{1}{\varsigma_{2}-\varsigma_{1}} \int_{\varsigma_{1}}^{\varsigma_{2}} \eta(\lambda) d \lambda \leq \frac{\eta\left(\varsigma_{1}\right)+\eta\left(\varsigma_{2}\right)}{2}
$$

for all $\varsigma_{1}, \varsigma_{2} \in\left[\varsigma_{1}, \varsigma_{2}\right]_{\mathcal{T}}$ with $\varsigma_{1}<\varsigma_{2}$, the double inequality referred to as the Hermite-Hadamard inequality for convex function and the constant $\frac{1}{2}$ is sharp, in the sense that $\frac{1}{2}$ cannot be replaced by a similar constant.

Since the publications of the above result in 1883, numerous papers with new evidence, different generalizations and developments have shown up in the literature, see References [26-31]. Recently, time scale versions of several inequalities such as Hermite-Hadamard inequality, Ostrowski inequality and Hardy-type inequalities are investigated by $[3,4,7,11]$.

This article presents several dynamical variants that are essentially based on Hermite-Hadamard inequality. Many of these variants are called the Hermite-Hadamard type. In the present assertion, Hermite-Hadamard inequalities for two non-negative auxiliary functions $\Psi_{1}$ and $\Psi_{2}$, which we attended on are quite exclusive from the existing ones. Finally, from an application perspective, the results are relevant to research for special means. Some unique instances also are mentioned which can be deduced from our consequences.

\section{Preliminaries}

A non-empty closed subset $\mathcal{R}$ of $\mathcal{T}$ is known as the time scale. The set of real numbers $\mathcal{R}$ and the integers $\mathcal{Z}$ are two well-known examples of time scales theory. In this sequel, we will mention $\mathcal{T}$, a time scale and $\left[\varsigma_{1}, \varsigma_{2}\right]_{\mathcal{T}}$, is a time-scaled interval. we want the idea of jump operators.

The symbol $\diamond$ denotes the forward jump operator and $\varrho$ is the backward jump operator are said through the formulas:

$$
\diamond(r)=\inf \{\lambda \in \mathcal{T}: \lambda>r\} \in \mathcal{T}, \quad \varrho(\omega)=\sup \{\lambda \in \mathcal{T}: \lambda<\omega\} \in \mathcal{T}
$$

We assemble as:

$$
\inf \varnothing:=\sup \mathcal{T}, \quad \sup \varnothing:=\inf \mathcal{T}
$$

The term $r$ is refer to be right-scattered if $\diamond(r)>r$ and $\varrho(\omega)<\omega$, then $\omega$ is refer to be left-scattered. The factors which are probably simultaneously proper-scattered and left-scattered are known as isolated. If $\diamond(r)=r$, then $r$ is said to be right dense, and if, $\varrho(\omega)=\omega$ then $\omega$ is said to be left dense. Moreover, the points $r, \omega$ are said to be dense if they are simultaneously right-dense and left-dense.

The mappings $\phi, \psi: \mathcal{T} \rightarrow[0,+\infty)$ defined by

$$
\phi(r):=\diamond(r)-r
$$




$$
\psi(r):=r-\varrho(r)
$$

are called the forward and backward graininess functions, respectively.

Definition 1. Let $\eta: \mathcal{T} \rightarrow \mathcal{R}$ be a real-valued function. Then $\eta$ is said to be $\mathcal{R} \mathcal{D}$-continuous on $\mathcal{R}$ if its left limit at any left dense point of $\mathcal{T}$ is finite and it is continuous on every right dense point of $\mathcal{T}$. All $\mathcal{R} \mathcal{D}$-continuous functions are denoted by $\mathbb{C}_{\mathcal{R D}}$.

Definition 2. A function $\mathcal{F}: \mathcal{T} \rightarrow \mathcal{R}$ is called a delta antiderivative of $\eta: \mathcal{T} \rightarrow \mathcal{R}$ if $\mathcal{F}^{\Delta}(r)=\eta(r)$, for all $r \in \mathcal{T}^{k}$. Then, one defines the delta integral by $\int_{\zeta_{1}}^{r} \eta(s) \Delta s=\mathcal{F}(r)-\mathcal{F}\left(\varsigma_{1}\right)$.

Theorem 1 ([2]). If $\eta \in \mathbb{C}_{R D}$ and $r \in \mathcal{T}^{k}$, then

$$
\int_{r}^{\diamond(r)} \eta(s) \Delta s=\phi(r) \eta(r) .
$$

Theorem 2 ([2]). Let $\varsigma_{1}, \varsigma_{2}, \varsigma_{3} \in \mathcal{T}, \beta \in \mathcal{R}$ and $\eta, \omega \in \mathbb{C}_{R D}$, then

(i) $\int_{\zeta_{1}}^{\varsigma_{2}}\left(\eta_{1}(\lambda)+\eta_{2}(\lambda)\right) \Delta \lambda=\int_{\zeta_{1}}^{\varsigma_{2}} \eta_{1}(\lambda) \Delta \lambda+\int_{\zeta_{1}}^{\varsigma_{2}} \eta_{2}(\lambda) \Delta \lambda$;

(ii) $\int_{\zeta_{1}}^{\varsigma_{2}} \beta \eta(\lambda) \Delta \lambda=\beta \int_{\zeta_{1}}^{\varsigma_{2}} \eta(\lambda) \Delta \lambda$;

(iii) $\int_{\varsigma_{1}}^{\varsigma_{2}} \eta(\lambda) \Delta \lambda=-\int_{\varsigma_{2}}^{\varsigma_{1}} \eta(\lambda) \Delta \lambda$;

(iv) $\int_{\varsigma_{1}}^{\varsigma_{2}} \eta(\lambda) \Delta \lambda=\int_{\varsigma_{1}}^{\varsigma_{3}} \eta(\lambda) \Delta \lambda+\int_{\varsigma_{3}}^{\varsigma_{2}} \eta(\lambda) \Delta \lambda$

(v) $\int_{\varsigma_{1}}^{\varsigma_{2}} \eta_{1}^{\diamond}(\lambda) \eta_{2}^{\Delta} \Delta \lambda=\left(\eta_{1} \eta_{2}\right)\left(\varsigma_{2}\right)-\left(\eta_{1} \eta_{2}\right)\left(\varsigma_{1}\right)-\int_{\varsigma_{1}}^{\varsigma_{2}} \eta_{1}^{\Delta}(\lambda) \eta_{2}(\lambda) \Delta(\lambda) ;$

(vi) $\int_{\zeta_{1}}^{\varsigma_{2}} \eta_{1}(\lambda) \eta_{2}^{\Delta} \Delta \lambda=\left(\eta_{1} \eta_{2}\right)\left(\varsigma_{2}\right)-\left(\eta_{1} \eta_{2}\right)\left(\varsigma_{1}\right)-\int_{\varsigma_{1}}^{\varsigma_{2}} \eta_{1}^{\Delta}(\lambda) \eta_{2}^{\diamond}(\lambda) \Delta(\lambda)$;

(vii) $\int_{\zeta_{1}}^{\varsigma_{2}} \eta(\lambda) \Delta(\lambda)=0$

(viii) If $\eta(\lambda) \geq 0$ for all $\lambda$, then $\int_{\zeta_{1}}^{\varsigma_{2}} \eta(\lambda) \Delta(\lambda) \geq 0$.

(ix) If $\left|\eta_{1}(\lambda)\right| \leq \eta_{2}(\lambda)$ on $\left[\varsigma_{1}, \varsigma_{2}\right]$, then $\left|\int_{\varsigma_{1}}^{\varsigma_{2}} \eta_{1}(\lambda) \Delta \lambda\right| \leq \int_{\varsigma_{1}}^{\varsigma_{2}} \eta_{2}(\lambda) \Delta(\lambda)$.

From assertion $(i x)$ of Theorem 2 for $\eta_{2}(\lambda)=\left|\eta_{1}(\lambda)\right|$ on $\left[\varsigma_{1}, \varsigma_{2}\right]$, we have

$$
\left|\int_{\varsigma_{1}}^{\varsigma_{2}} \eta(\lambda) \Delta \lambda\right| \leq \int_{\varsigma_{1}}^{\varsigma_{2}}|\eta(\lambda)| \Delta(\lambda) .
$$

The following example exhibits some practice related to the real world on time scale analysis.

Example 1. Let $\mathcal{N}(r)$ be the range of plant life of one specific type at time $r$ in a certain vicinity. By experiments, we recognize that $\mathcal{N}$ grows exponentially consistent with $\mathcal{N}^{\prime}=\mathcal{N}$ during the months of May to August. At the 
beginning of September, all vegetation abruptly dies; however, the seeds remain in the ground and begin growing again at the start of May with $\mathcal{N}$ now being doubled. We model this case using the time scale

$$
\mathcal{T}=\bigcup_{\lambda=0}^{\infty}[2 \lambda, 2 \lambda+1]
$$

where $r=0$ is 1 May of the current year, $r=1$ is 1 September of the current year, $r=2$ is 1 May of the next year, $r=3$ is 1 September of the subsequent year, and so on. we have

$$
\delta(\lambda)=\left\{\begin{array}{ccc}
0, & \text { if } & 2 \lambda \leq r<2 \lambda+1 \\
1, & \text { if } & r=2 \lambda+1
\end{array}\right.
$$

On $[2 \lambda, 2 \lambda+1]$ we have $\mathcal{N}^{\prime}=\mathcal{N}$ that is, $\mathcal{N}^{\Delta}=\mathcal{N}$. Therefore, we have $\mathcal{N}(2 \lambda+2)=2 \mathcal{N}(2 \lambda+1)$; that is, $\Delta \mathcal{N}(2 \lambda+1)=\mathcal{N}(2 \lambda+1)$ that is, $\mathcal{N} \Delta=\mathcal{N}$ at $2 \lambda+1$. As a result, $\mathcal{N}$ is a solution of the dynamic equation

$$
\mathcal{N}^{\Delta}=\mathcal{N}
$$

Thus, if $\mathcal{N}(0)=1$ is given, $\mathcal{N}$ is exactly $e_{1}(., 0)$ on the time scale $\mathcal{T}$.

We now consider a class of convex function with respect to two arbitrary functions $\Psi_{1}$ and $\Psi_{2}$ on time scale $\mathcal{T}$ and associated notions.

Definition 3. Consider a time scale $\mathcal{T}$ and let $\Psi_{1}, \Psi_{2}:(0,1) \subseteq J \rightarrow \mathcal{R}$ be two nonnegative functions. A function $\eta: \mathrm{Y}=\left[\varsigma_{1}, \varsigma_{2}\right]_{\mathcal{T}} \rightarrow \mathcal{R}$ is said to be a $\left(\Psi_{1}, \Psi_{2}\right)$-convex function with respect to two nonnegative functions $\Psi_{1}$ and $\Psi_{2}$ if

$$
\eta\left((1-r) \varsigma_{1}+r \varsigma_{2}\right) \leq \Psi_{1}(1-r) \Psi_{2}(r) \eta\left(\varsigma_{1}\right)+\Psi_{2}(1-r) \Psi_{1}(r) \eta\left(\varsigma_{2}\right), \quad \forall \varsigma_{1}, \varsigma_{2} \in \mathrm{Y}, r \in[0,1] .
$$

We now discuss several special cases of Definition 3.

(I). If $\Psi_{1}(r)=\Psi_{2}(r)=r^{s}$ in Definition 3, then we attain Breckner type of s-convex functions.

Definition 4. Consider a time scale $\mathcal{T}$ and $s \in[0,1]$ be a real number. We say that $\eta: Y=\left[\varsigma_{1}, \varsigma_{2}\right] \mathcal{T} \rightarrow \mathcal{R}$ is a Breckner type s-convex function, if

$$
\eta\left((1-r) \varsigma_{1}+r \varsigma_{2}\right) \leq(1-r)^{s^{s}} r^{s}\left[\eta\left(\varsigma_{1}\right)+\eta\left(\varsigma_{2}\right)\right], \quad \forall \varsigma_{1}, \varsigma_{2} \in \mathrm{Y}, r \in[0,1] .
$$

(II). If $\Psi_{1}(r)=\Psi_{2}(r)=1$ in Definition 3, then we have $P$-convex function.

Definition 5. Consider a time scale $\mathcal{T}$. We say that $\eta: \mathrm{Y}=\left[\varsigma_{1}, \varsigma_{2}\right] \mathcal{T} \rightarrow \mathcal{R}$ is a P-convex function, if

$$
\eta\left((1-r) \varsigma_{1}+r \varsigma_{2}\right) \leq\left[\eta\left(\varsigma_{1}\right)+\eta\left(\varsigma_{2}\right)\right], \quad \forall \varsigma_{1}, \varsigma_{2} \in \mathrm{Y} .
$$

Remark 1. For exceptional appropriate selections of functions $\Psi_{1}$ and $\Psi_{2}$, one can find many novel and existing classes of convex functions as special cases. See, for example, References [11,16-23,32,33]. This presents that the idea of $\left(\Psi_{1}, \Psi_{2}\right)$-convex function is quite a general and unifying one.

Definition 6. ([3]). Let $\chi_{k}: \mathcal{T}^{2} \rightarrow \mathcal{R}, k \in \mathcal{N}_{0}$ be defined by

$$
\chi_{0}(r, \omega)=1 \forall r, \omega \in \mathcal{T}
$$


and then recursively by

$$
\chi_{k+1}(r, \omega)=\int_{\omega}^{r} \chi_{k}(\tau, \omega) \Delta \tau
$$

for all $\omega, r \in \mathcal{T}$.

In [11], Tahir et al. obtained the subsequent Lemma's:

Lemma 1 ([11]). Let $\eta: \mathcal{T} \rightarrow \mathcal{R}$ be a differentiable mapping and $\varsigma_{1}<\varsigma_{2} \in \mathcal{T}$. Let $\eta^{\Delta} \in \mathbb{C}_{R D}$, then

$$
\begin{aligned}
& \eta\left(\varsigma_{1}\right)\left\{1-\chi_{2}(1,0)\right\}+\eta\left(\varsigma_{2}\right) \chi_{2}(1,0)-\frac{1}{\varsigma_{2}-\varsigma_{1}} \int_{\zeta_{1}}^{\varsigma_{2}} \eta^{\diamond}(\lambda) \Delta \lambda \\
& =\frac{\varsigma_{2}-\varsigma_{1}}{2} \int_{0}^{1} \int_{0}^{1}\left\{\eta^{\Delta}\left(r \varsigma_{1}+(1-r) \varsigma_{2}\right)-\eta^{\Delta}\left(\omega \varsigma_{1}+(1-\omega) \varsigma_{2}\right)\right\}(r-\omega) \Delta r \Delta \omega .
\end{aligned}
$$

Lemma 2 ([11]). Let $\eta:\left[\varsigma_{1}, \varsigma_{2}\right] \mathcal{T} \subseteq \mathcal{T} \rightarrow \mathcal{R}$ be a delta differentiable mapping on $\mathcal{T}^{\circ}$ and $\varsigma_{1}<\varsigma_{2} \in \mathcal{T}$. Let $\eta^{\Delta} \in \mathbb{C}_{R D}$, then the following inequalities holds:

$$
\begin{aligned}
& \eta\left(\frac{\varsigma_{1}+\varsigma_{2}}{2}\right)-\frac{1}{\varsigma_{2}-\varsigma_{1}} \int_{\varsigma_{1}}^{\varsigma_{2}} \eta^{\diamond}(\lambda) \Delta \lambda \\
& =\frac{\varsigma_{2}-\varsigma_{1}}{2} \int_{0}^{1} \int_{0}^{1}\left[\eta^{\Delta}\left(r \varsigma_{1}+(1-r) \varsigma_{2}\right)-\eta^{\Delta}\left(\omega \varsigma_{1}+(1-\omega) \varsigma_{2}\right)\right](\varphi(\omega)-\varphi(r)) \Delta r \Delta \omega,
\end{aligned}
$$

where

$$
\varphi(\lambda)=\left\{\begin{array}{cc}
\lambda, & \lambda \in\left[0, \frac{1}{2}\right] \\
\lambda-1, & \lambda \in\left(\frac{1}{2}, 1\right]
\end{array}\right.
$$

\section{Main Results}

The subsequent dynamic identity performs a key function in inaugurating the main consequences of this paper. The identification is expressed as follows.

Lemma 3. Consider a time scale $\mathcal{T}$ and $\mathrm{Y}=\left[\varsigma_{1}, \varsigma_{2}\right]_{\mathcal{T}} \subseteq \mathcal{T}$ such that $\varsigma_{1}<\varsigma_{2}$ and $\varsigma_{1}, \varsigma_{2} \in \mathcal{T}$. Assume that there is a delta differentiable function $\eta: \mathrm{Y} \rightarrow \mathcal{R}$ on $\mathrm{Y}^{\circ}\left(\mathrm{Y}^{\circ}\right.$ is the interior of $\left.\mathrm{Y}\right)$. If $\eta^{\Delta} \in \mathbb{C}_{\mathcal{R} \mathcal{D}}$, then

$$
\begin{aligned}
& \frac{\eta\left(\varsigma_{1}\right)+\eta\left(\varsigma_{2}\right)}{2}-\frac{1}{\varsigma_{2}-\varsigma_{1}} \int_{\zeta_{1}}^{\varsigma_{2}} \eta^{\diamond}(\lambda) \Delta \lambda \\
& =\frac{1}{2\left(\varsigma_{2}-\varsigma_{1}\right)}\left[\int_{\zeta_{1}}^{\varsigma_{2}}\left(\lambda-\varsigma_{1}\right) \eta^{\Delta}(\lambda) \Delta \lambda-\int_{\zeta_{1}}^{\varsigma_{2}}\left(\varsigma_{2}-\lambda\right) \eta^{\Delta}(\lambda) \Delta \lambda\right] .
\end{aligned}
$$

Proof. Using assertion (vi) from Theorem 2 and taking into consideration the proof of Lemma 3.1 in [3], we have

$$
\int_{\varsigma_{1}}^{\varsigma_{2}} \eta_{1}(\lambda) \eta_{2}^{\Delta}(\lambda) \Delta \lambda=\left(\eta_{1} \eta_{2}\right)\left(\varsigma_{2}\right)-\left(\eta_{1} \eta_{2}\right)\left(\varsigma_{1}\right)-\int_{\varsigma_{1}}^{\varsigma_{2}} \eta_{1}^{\Delta}(\lambda) \eta_{2}^{\diamond}(\lambda) \Delta \lambda
$$


with $\eta_{1}(\lambda)=\frac{\lambda-\varsigma_{1}}{\varsigma_{2}-\zeta_{1}}, \eta_{2}(\lambda)=\eta(\lambda)$ in first integral and $\eta_{1}(\lambda)=\frac{\lambda-\varsigma_{2}}{\varsigma_{1}-\varsigma_{2}}, \eta_{2}(\lambda)=\eta(\lambda)$ in second integral, we have

$$
\begin{aligned}
& \int_{\varsigma_{1}}^{\varsigma_{2}} \frac{\lambda-\varsigma_{1}}{\varsigma_{2}-\varsigma_{1}} \eta^{\Delta}(\lambda) \Delta(\lambda)-\int_{\varsigma_{1}}^{\varsigma_{2}} \frac{\lambda-\varsigma_{2}}{\varsigma_{1}-\varsigma_{2}} \eta^{\Delta}(\lambda) \Delta(\lambda) \\
& =\left[\eta\left(\varsigma_{2}\right)-\frac{1}{\varsigma_{2}-\varsigma_{2}} \int_{\varsigma_{1}}^{\varsigma_{2}} \eta^{\diamond}(\lambda) \Delta \lambda\right]-\left[-\eta\left(\varsigma_{1}\right)+\frac{1}{\varsigma_{2}-\varsigma_{2}} \int_{\zeta_{1}}^{\varsigma_{2}} \eta \diamond(\lambda) \Delta \lambda\right] \\
& =\eta\left(\varsigma_{1}\right)+\eta\left(\varsigma_{2}\right)-\frac{2}{\varsigma_{2}-\varsigma_{2}} \int_{\varsigma_{1}}^{\varsigma_{2}} \eta^{\diamond}(\lambda) \Delta \lambda .
\end{aligned}
$$

By taking product on both sides of (12) with $\frac{1}{2}$, we get the desired identity (2).

Corollary 1. Under the assumptions of Lemma 3, we have

$$
\begin{aligned}
& \frac{\eta\left(\varsigma_{1}\right)+\eta\left(\varsigma_{2}\right)}{2}-\frac{1}{\varsigma_{2}-\varsigma_{1}} \int_{\varsigma_{1}}^{\varsigma_{2}} \eta^{\diamond}(\lambda) d \lambda \\
& =\frac{\varsigma_{2}-\varsigma_{1}}{2}\left[\int_{0}^{1} r \eta^{\Delta}\left(r \varsigma_{2}+(1-r) \varsigma_{1}\right) \Delta r-\int_{0}^{1} r \eta^{\Delta}\left(r \varsigma_{1}+(1-r) \varsigma_{2}\right) \Delta r\right] .
\end{aligned}
$$

Proof. Using Lemma 3 and making the change of variable technique, i.e, by putting $r=\frac{\lambda-\varsigma_{1}}{\varsigma_{2}-\varsigma_{1}}$, we obtain

$$
\int_{\varsigma_{1}}^{\varsigma_{2}} \frac{\lambda-\varsigma_{1}}{\varsigma_{2}-\varsigma_{1}} \eta^{\Delta}(\lambda) \Delta(\lambda)=\left(\varsigma_{2}-\varsigma_{1}\right) \int_{0}^{1} r \eta^{\Delta}\left(r \varsigma_{2}+(1-r) \varsigma_{1}\right) \Delta r
$$

Analogously:

$$
\int_{\varsigma_{1}}^{\varsigma_{2}} \frac{\lambda-\varsigma_{2}}{\varsigma_{2}-\varsigma_{1}} \eta^{\Delta}(\lambda) \Delta(\lambda)=\left(\varsigma_{2}-\varsigma_{1}\right) \int_{0}^{1} r \eta^{\Delta}\left(r \varsigma_{1}+(1-r) \varsigma_{2}\right) \Delta r .
$$

Suitable rearrangements complete the proof.

Corollary 2. Letting $\mathcal{T}=\mathcal{R}$ and making change of variable on right hand side of Lemma 3, then we attain the following result as a Lemma 2.1 in [34].

$$
\frac{\eta\left(\varsigma_{1}\right)+\eta\left(\varsigma_{2}\right)}{2}-\frac{1}{\varsigma_{2}-\varsigma_{1}} \int_{\varsigma_{1}}^{\varsigma_{2}} \eta(\lambda) d \lambda=\frac{1}{2\left(\varsigma_{2}-\varsigma_{1}\right)}\left[\int_{\varsigma_{1}}^{\varsigma_{2}}\left(\lambda-\varsigma_{1}\right) \eta^{\prime}(\lambda) \Delta \lambda-\int_{\varsigma_{1}}^{\varsigma_{2}}\left(\varsigma_{2}-\lambda\right) \eta^{\prime}(\lambda) \Delta \lambda\right] .
$$

Corollary 3. If $\mathcal{T}=\mathcal{N}$, then under the assumption of Lemma 3 and assume that $\varsigma_{1}=0, \varsigma_{2}=\delta, \lambda=\varepsilon$ and $\eta(\kappa)=\lambda_{\kappa}$, then

$$
\frac{\lambda_{0}+\lambda_{1}}{2}-\frac{1}{\delta} \sum_{\varepsilon=0}^{\delta} \lambda_{\varepsilon}=\frac{1}{2 \delta}\left[\sum_{\varepsilon=0}^{\delta-1} \varepsilon \Delta \lambda_{\varepsilon}-\sum_{\varepsilon=0}^{\delta-1}(\delta-\varepsilon) \Delta \lambda_{\varepsilon}\right]
$$


Theorem 3. Consider a time scale $\mathcal{T}$ and $\mathrm{Y}=\left[\varsigma_{1}, \varsigma_{2}\right] \mathcal{T} \subseteq \mathcal{T}$ such that $\varsigma_{1}<\varsigma_{2}$ and $\varsigma_{1}, \varsigma_{2} \in \mathcal{T}$. Assume that there is a delta differentiable function $\eta: \mathrm{Y} \rightarrow \mathcal{R}$ on $\mathrm{Y}^{\circ}\left(\mathrm{Y}^{\circ}\right.$ is the interior of $\left.\mathrm{Y}\right)$. If $\left|\eta^{\Delta}\right|$ is $\left(\Psi_{1}, \Psi_{2}\right)$-convex, then

$$
\begin{aligned}
& \left|\frac{\eta\left(\varsigma_{1}\right)+\eta\left(\varsigma_{2}\right)}{2}-\frac{1}{\varsigma_{2}-\varsigma_{1}} \int_{\varsigma_{1}}^{\varsigma_{2}} \eta^{\diamond}(\lambda) \Delta \lambda\right| \\
& \leq \frac{\varsigma_{2}-\varsigma_{1}}{2}\left[\Omega^{*}(r)+\Lambda^{*}(r)\right]\left\{\left|\eta^{\Delta}\left(\varsigma_{1}\right)\right|+\left|\eta^{\Delta}\left(\varsigma_{2}\right)\right|\right\},
\end{aligned}
$$

where

$$
\Omega^{*}(r)=\int_{0}^{1} r \Psi_{1}(r) \Psi_{2}(1-r) \Delta r
$$

and

$$
\Lambda^{*}(r)=\int_{0}^{1} r \Psi_{2}(r) \Psi_{1}(1-r) \Delta r
$$

Proof. Using Corollary 1, property of modulus and $\left(\Psi_{1}, \Psi_{2}\right)$-convexity of $\left|\eta^{\Delta}\right|$ we get

$$
\begin{aligned}
& \left|\frac{\eta\left(\varsigma_{1}\right)+\eta\left(\varsigma_{2}\right)}{2}-\frac{1}{\varsigma_{2}-\varsigma_{1}} \int_{\varsigma_{1}}^{\varsigma_{2}} \eta^{\diamond}(\lambda) \Delta \lambda\right| \\
& \leq \frac{\varsigma_{2}-\varsigma_{1}}{2}\left[\int_{0}^{1} r\left|\eta^{\Delta}\left(r_{\zeta_{2}}+(1-r) \varsigma_{1}\right)\right| \Delta r+\int_{0}^{1} r\left|\eta^{\Delta}\left(r \varsigma_{1}+(1-r) \varsigma_{2}\right)\right| \Delta r\right] \\
& \leq \frac{\varsigma_{2}-\varsigma_{1}}{2}\left[\int_{0}^{1} r\left\{\Psi_{1}(r) \Psi_{2}(1-r)\left|\eta^{\Delta}\left(\varsigma_{2}\right)\right|+\Psi_{2}(r) \Psi_{1}(1-r)\left|\eta^{\Delta}\left(\varsigma_{1}\right)\right|\right\} \Delta r\right. \\
& \left.\quad+\int_{0}^{1} r\left\{\Psi_{1}(r) \Psi_{2}(1-r)\left|\eta^{\Delta}\left(\varsigma_{1}\right)\right|+\Psi_{2}(r) \Psi_{1}(1-r)\left|\eta^{\Delta}\left(\varsigma_{2}\right)\right|\right\} \Delta r\right] \\
& =\frac{\varsigma_{2}-\varsigma_{1}}{2}\left[\Omega^{*}(r)+\Lambda^{*}(r)\right]\left\{\left|\eta^{\Delta}\left(\varsigma_{1}\right)\right|+\left|\eta^{\Delta}\left(\varsigma_{2}\right)\right|\right\},
\end{aligned}
$$

which is the required result.

On an arbitrary time scale of $\mathcal{T}$, we may observe that the variant demonstrated above is a speculation of some new outcomes. We now discuss some new special cases which can be derived directly from Theorem 3.

I. Letting $\Psi_{1}(r)=\Psi_{2}(r)=r^{s}$, then we have s-convex functions of Breckner type.

Corollary 4. Consider a time scale $\mathcal{T}$ and $\mathrm{Y}=\left[\varsigma_{1}, \varsigma_{2}\right] \mathcal{T} \subseteq \mathcal{T}$ such that $\varsigma_{1}<\varsigma_{2}$ and $\varsigma_{1}, \varsigma_{2} \in \mathcal{T}$. Assume that there is a delta differentiable function $\eta: \mathrm{Y} \rightarrow \mathcal{R}$ on $\mathrm{Y}^{\circ}\left(\mathrm{Y}^{\circ}\right.$ is the interior of $\left.\mathrm{Y}\right)$. If $\left|\eta^{\Delta}\right|$ is a Breakner type s-convex function, then

$$
\left|\frac{\eta\left(\varsigma_{1}\right)+\eta\left(\varsigma_{2}\right)}{2}-\frac{1}{\varsigma_{2}-\varsigma_{1}} \int_{\varsigma_{1}}^{\varsigma_{2}} \eta^{\diamond}(\lambda) \Delta \lambda\right| \leq\left(\varsigma_{2}-\varsigma_{1}\right) \mathcal{H}_{1}(r)\left\{\left|\eta^{\Delta}\left(\varsigma_{1}\right)\right|+\left|\eta^{\Delta}\left(\varsigma_{2}\right)\right|\right\},
$$


where

$$
\mathcal{H}_{1}(r)=\int_{0}^{1} r^{s+1}(1-r)^{s} \Delta r
$$

II. Letting $\Psi_{1}(r)=\Psi_{2}(r)=1$, then we have $P$-convex functions.

Corollary 5. Consider a time scale $\mathcal{T}$ and $\mathrm{Y}=\left[\varsigma_{1}, \varsigma_{2}\right] \mathcal{T} \subseteq \mathcal{T}$ such that $\varsigma_{1}<\varsigma_{2}$ and $\varsigma_{1}, \varsigma_{2} \in \mathcal{T}$. Assume that there is a delta differentiable function $\eta: \mathrm{Y} \rightarrow \mathcal{R}$ on $\mathrm{Y}^{\circ}\left(\mathrm{Y}^{\circ}\right.$ is the interior of $\left.\mathrm{Y}\right)$. If $\left|\eta^{\Delta}\right|$ is a P-convex function, then

$$
\left|\frac{\eta\left(\varsigma_{1}\right)+\eta\left(\varsigma_{2}\right)}{2}-\frac{1}{\varsigma_{2}-\varsigma_{1}} \int_{\varsigma_{1}}^{\varsigma_{2}} \eta^{\diamond}(\lambda) \Delta \lambda\right| \leq \frac{\left(\varsigma_{2}-\varsigma_{1}\right)}{4}\left\{\left|\eta^{\Delta}\left(\varsigma_{1}\right)\right|+\left|\eta^{\Delta}\left(\varsigma_{2}\right)\right|\right\} .
$$

Remark 2. Letting $\mathcal{T}=\mathcal{R}$, then our delta integral is the reduces to the usual Riemann integral from calculus. Hence, Theorem 3 becomes

$$
\left|\frac{\eta\left(\varsigma_{1}\right)+\eta\left(\varsigma_{2}\right)}{2}-\frac{1}{\varsigma_{2}-\varsigma_{1}} \int_{\varsigma_{1}}^{\varsigma_{2}} \eta(\lambda) \Delta \lambda\right| \leq \frac{\varsigma_{2}-\varsigma_{1}}{2}\left[\Omega^{*}(r)+\Lambda^{*}(r)\right]\left\{\left|\eta^{\prime}\left(\varsigma_{1}\right)\right|+\left|\eta^{\prime}\left(\varsigma_{2}\right)\right|\right\} .
$$

where

$$
\Omega^{*}(r)=\int_{0}^{1} r \Psi_{1}(r) \Psi_{2}(1-r) d r
$$

and

$$
\Lambda^{*}(r)=\int_{0}^{1} r \Psi_{2}(r) \Psi_{1}(1-r) d r
$$

Remark 3. Letting $\mathcal{T}=\mathcal{R}$ along with $\Psi_{1}(r)=r^{6}$ and $\Psi_{2}(r)=1$, then Theorem 3 becomes Theorem 2.2 in [34].

Theorem 4. Consider a time scale $\mathcal{T}$ and $\mathrm{Y}=\left[\varsigma_{1}, \varsigma_{2}\right] \mathcal{T} \subseteq \mathcal{T}$ such that $\varsigma_{1}<\varsigma_{2}$ and $\varsigma_{1}, \varsigma_{2} \in \mathcal{T}$. Assume that there is a delta differentiable function $\eta: \mathrm{Y} \rightarrow \mathcal{R}$ on $\mathrm{Y}^{\circ}\left(\mathrm{Y}^{\circ}\right.$ is the interior of $\left.\mathrm{Y}\right)$. If $\left|\eta^{\Delta}\right|^{q}$ is $\left(\Psi_{1}, \Psi_{2}\right)$-convex, for $\alpha>1$ such that $\frac{1}{\alpha}+\frac{1}{\beta}=1$, then

$$
\begin{aligned}
& \left|\frac{\eta\left(\varsigma_{1}\right)+\eta\left(\varsigma_{2}\right)}{2}-\frac{1}{\varsigma_{2}-\varsigma_{1}} \int_{\varsigma_{1}}^{\varsigma_{2}} \eta^{\diamond}(\lambda) \Delta \lambda\right| \\
& \leq \frac{\varsigma_{2}-\varsigma_{1}}{2}\left(\int_{0}^{1} r^{\alpha} \Delta r\right)^{\frac{1}{\alpha}}\left[\left(\int_{0}^{1}\left\{\Psi_{1}(r) \Psi_{2}(1-r)\left|\eta^{\Delta}\left(\varsigma_{2}\right)\right|^{\beta}+\Psi_{2}(r) \Psi_{1}(1-r)\left|\eta^{\Delta}\left(\varsigma_{1}\right)\right|^{\beta}\right\}\right)^{\frac{1}{\beta}}\right. \\
& \left.\quad+\left(\int_{0}^{1}\left\{\Psi_{1}(r) \Psi_{2}(1-r)\left|\eta^{\Delta}\left(\varsigma_{1}\right)\right|^{\beta}+\Psi_{2}(r) \Psi_{1}(1-r)\left|\eta^{\Delta}\left(\varsigma_{2}\right)\right|^{\beta}\right\}\right)^{\frac{1}{\beta}}\right] .
\end{aligned}
$$


Proof. Using Corollary 3, property of modulus, Hölder's integral inequality and ( $\left.\Psi_{1}, \Psi_{2}\right)$-convexity of $\left|\eta^{\Delta}\right|$, we obtain

$$
\begin{aligned}
& \left|\frac{\eta\left(\varsigma_{1}\right)+\eta\left(\varsigma_{2}\right)}{2}-\frac{1}{\varsigma_{2}-\varsigma_{1}} \int_{\varsigma_{1}}^{\varsigma_{2}} \eta^{\diamond}(\lambda) \Delta \lambda\right| \\
& \leq \frac{\varsigma_{2}-\varsigma_{1}}{2}\left|\int_{0}^{1} r \eta^{\Delta}\left(r \varsigma_{2}+(1-r) \varsigma_{1}\right) \Delta r-\int_{0}^{1} r \eta^{\Delta}\left(r \varsigma_{1}+(1-r) \varsigma_{2}\right) \Delta r\right| \\
& \leq \frac{\varsigma_{2}-\varsigma_{1}}{2}\left|\int_{0}^{1} r \eta^{\Delta}\left(r \varsigma_{2}+(1-r) \varsigma_{1}\right) \Delta r\right|+\left|\int_{0}^{1} r \eta^{\Delta}\left(r \varsigma_{1}+(1-r) \varsigma_{2}\right) \Delta r\right| \\
& \leq \frac{\varsigma_{2}-\varsigma_{1}}{2}\left(\int_{0}^{1} r^{\alpha} \Delta r\right)^{\frac{1}{\alpha}}\left[\left(\int_{0}^{1}\left|\eta^{\Delta}\left(r \zeta_{2}+(1-r) \varsigma_{1}\right)\right|^{\beta}\right)^{\frac{1}{\beta}}+\left(\int_{0}^{1}\left|\eta^{\Delta}\left(r \zeta_{1}+(1-r) \varsigma_{2}\right)\right|^{\beta}\right)^{\frac{1}{\beta}}\right] \\
& \leq \frac{\varsigma_{2}-\varsigma_{1}}{2}\left(\int_{0}^{1} r^{\alpha} \Delta r\right)^{\frac{1}{\alpha}}\left[\left(\int_{0}^{1}\left\{\Psi_{1}(r) \Psi_{2}(1-r)\left|\eta^{\Delta}\left(\varsigma_{2}\right)\right|^{\beta}+\Psi_{2}(r) \Psi_{1}(1-r)\left|\eta^{\Delta}\left(\varsigma_{1}\right)\right|^{\beta}\right\}\right)^{\frac{1}{\beta}}\right. \\
& \left.\quad+\left(\int_{0}^{1}\left\{\Psi_{1}(r) \Psi_{2}(1-r)\left|\eta^{\Delta}\left(\varsigma_{1}\right)\right|^{\beta}+\Psi_{2}(r) \Psi_{1}(1-r)\left|\eta^{\Delta}\left(\varsigma_{2}\right)\right|^{\beta}\right\}\right)^{\frac{1}{\beta}}\right]
\end{aligned}
$$

which is the required result.

In the following, we give two corollaries that follow from the special cases of Theorem 4.

I. Letting $\Psi_{1}(r)=\Psi_{2}(r)=r^{s}$, then we have s-convex functions of Breckner type.

Corollary 6. Consider a time scale $\mathcal{T}$ and $\mathrm{Y}=\left[\varsigma_{1}, \varsigma_{2}\right] \mathcal{T} \subseteq \mathcal{T}$ such that $\varsigma_{1}<\varsigma_{2}$ and $\varsigma_{1}, \varsigma_{2} \in \mathcal{T}$. Assume that there is a delta differentiable function $\eta: \mathrm{Y} \rightarrow \mathcal{R}$ on $\mathrm{Y}^{\circ}\left(\mathrm{Y}^{\circ}\right.$ is the interior of $\left.\mathrm{Y}\right)$. If $\left|\eta^{\Delta}\right|^{q}$ where $\alpha>1, \frac{1}{\alpha}+\frac{1}{\beta}=1$, is Breckner type of a s-convex, then

$$
\left|\frac{\eta\left(\varsigma_{1}\right)+\eta\left(\varsigma_{2}\right)}{2}-\frac{1}{\varsigma_{2}-\varsigma_{1}} \int_{\varsigma_{1}}^{\varsigma_{2}} \eta^{\diamond}(\lambda) \Delta \lambda\right| \leq\left(\varsigma_{2}-\varsigma_{1}\right)\left(\int_{0}^{1} r^{\alpha} \Delta r\right)^{\frac{1}{\alpha}}\left(\mathcal{H}_{2}(r)\right)^{\frac{1}{\beta}}\left\{\left|\eta^{\Delta}\left(\varsigma_{1}\right)\right|^{\beta}+\left|\eta^{\Delta}\left(\varsigma_{2}\right)\right|^{\beta}\right\}^{\frac{1}{\beta}},
$$

where

$$
\mathcal{H}_{2}(r)=\int_{0}^{1} r^{s}(1-r)^{s} \Delta r
$$

II. Letting $\Psi_{1}(r)=\Psi_{2}(r)=1$, then we have $P$-convex functions.

Corollary 7. Consider a time scale $\mathcal{T}$ and $\mathrm{Y}=\left[\varsigma_{1}, \varsigma_{2}\right] \mathcal{T} \subseteq \mathcal{T}$ such that $\varsigma_{1}<\varsigma_{2}$ and $\varsigma_{1}, \varsigma_{2} \in \mathcal{T}$. Assume that there is a delta differentiable function $\eta: \mathrm{Y} \rightarrow \mathcal{R}$ on $\mathrm{Y}^{\circ}\left(\mathrm{Y}^{\circ}\right.$ is the interior of $\left.\mathrm{Y}\right)$. If $\left|\eta^{\Delta}\right|^{q}$ where $\alpha>1, \frac{1}{\alpha}+\frac{1}{\beta}=1$, is a P-convex function, then

$$
\left|\frac{\eta\left(\varsigma_{1}\right)+\eta\left(\varsigma_{2}\right)}{2}-\frac{1}{\varsigma_{2}-\varsigma_{1}} \int_{\varsigma_{1}}^{\varsigma_{2}} \eta^{\diamond}(\lambda) \Delta \lambda\right| \leq \frac{\varsigma_{2}-\varsigma_{1}}{2}\left(\int_{0}^{1} r^{\alpha} \Delta r\right)^{\frac{1}{\alpha}}\left\{\left|\eta^{\Delta}\left(\varsigma_{1}\right)\right|^{\beta}+\left|\eta^{\Delta}\left(\varsigma_{2}\right)\right|^{\beta}\right\}^{\frac{1}{\beta}} .
$$


Remark 4. Letting $\mathcal{T}=\mathcal{R}$, then our delta integral reduces to the usual Riemann integral from calculus. Hence, Theorem 4 becomes

$$
\begin{aligned}
& \left|\frac{\eta\left(\varsigma_{1}\right)+\eta\left(\varsigma_{2}\right)}{2}-\frac{1}{\varsigma_{2}-\varsigma_{1}} \int_{\zeta_{1}}^{\varsigma_{2}} \eta(\lambda) d \lambda\right| \\
& \leq \frac{\varsigma_{2}-\varsigma_{1}}{2(\alpha+1)^{\frac{1}{\alpha}}}\left[\left(\int_{0}^{1}\left\{\Psi_{1}(r) \Psi_{2}(1-r)\left|\eta^{\prime}\left(\varsigma_{2}\right)\right|^{\beta}+\Psi_{2}(r) \Psi_{1}(1-r)\left|\eta^{\prime}\left(\varsigma_{1}\right)\right|^{\beta}\right\}\right)^{\frac{1}{\beta}}\right. \\
& \left.\quad+\left(\int_{0}^{1}\left\{\Psi_{1}(r) \Psi_{2}(1-r)\left|\eta^{\prime}\left(\varsigma_{1}\right)\right|^{\beta}+\Psi_{2}(r) \Psi_{1}(1-r)\left|\eta^{\prime}\left(\varsigma_{2}\right)\right|^{\beta}\right\}\right)^{\frac{1}{\beta}}\right]
\end{aligned}
$$

Remark 5. Letting $\mathcal{T}=\mathcal{R}$ along with $\Psi_{1}(r)=r^{6}$ and $\Psi_{2}(r)=1$, then Theorem 4 becomes Theorem 2.3 in [34].

Lemma 4. Consider a time scale $\mathcal{T}$ and $\mathrm{Y}=\left[\varsigma_{1}, \varsigma_{2}\right]_{\mathcal{T}} \subseteq \mathcal{T}$ such that $\varsigma_{1}<\varsigma_{2}$ and $\varsigma_{1}, \varsigma_{2} \in \mathcal{T}$. Assume that there is a delta differentiable function $\eta: \mathrm{Y} \rightarrow \mathcal{R}$ on $\mathrm{Y}^{\circ}\left(\mathrm{Y}^{\circ}\right.$ is the interior of $\left.\mathrm{Y}\right)$. If $\eta^{\Delta} \in \mathbb{C}_{\mathcal{R D}}$, then the following inequality holds:

$$
\begin{aligned}
& \eta\left(\frac{\varsigma_{1}+\varsigma_{2}}{2}\right)-\frac{1}{\varsigma_{2}-\varsigma_{1}} \int_{\varsigma_{1}}^{\varsigma_{2}} \eta^{\diamond}(\lambda) \Delta \lambda \\
& =\frac{1}{\left(\varsigma_{2}-\varsigma_{1}\right)}\left[\int_{\varsigma_{1}}^{\frac{\varsigma_{1}+\varsigma_{2}}{2}}\left(\lambda-\varsigma_{1}\right) \eta^{\Delta}(\lambda) \Delta \lambda+\int_{\frac{\varsigma_{1}+\varsigma_{2}}{2}}^{\varsigma_{2}}\left(\lambda-\varsigma_{2}\right) \eta^{\Delta}(\lambda) \Delta \lambda\right] .
\end{aligned}
$$

Proof. Using assertion (vi) from Theorem 2 and taking into consideration the proof of Lemma 3.1 in Reference [3], we have

$$
\int_{\varsigma_{1}}^{\varsigma_{2}} \eta_{1}(\lambda) \eta_{2}^{\Delta}(\lambda) \Delta \lambda=\left(\eta_{1} \eta_{2}\right)\left(\varsigma_{2}\right)-\left(\eta_{1} \eta_{2}\right)\left(\varsigma_{1}\right)-\int_{\varsigma_{1}}^{\varsigma_{2}} \eta_{1}^{\Delta}(\lambda) \eta_{2}^{\diamond}(\lambda) \Delta \lambda
$$

with $\eta_{1}(\lambda)=\frac{\lambda-\varsigma_{1}}{\varsigma_{2}-\varsigma_{1}}, \eta_{2}(\lambda)=\eta(\lambda)$ in first integral and $\eta_{1}(\lambda)=\frac{\lambda-\varsigma_{2}}{\varsigma_{1}-\varsigma_{2}}, \eta_{2}(\lambda)=\eta(\lambda)$ in second integral, we have

$$
\begin{aligned}
& \int_{\varsigma_{1}}^{\frac{\varsigma_{1}+\varsigma_{2}}{2}} \frac{\lambda-\varsigma_{1}}{\varsigma_{2}-\varsigma_{1}} \eta^{\Delta}(\lambda) \Delta(\lambda)+\int_{\frac{\varsigma_{1}+\varsigma_{2}}{2}}^{\varsigma_{2}} \frac{\lambda-\varsigma_{2}}{\varsigma_{1}-\varsigma_{2}} \eta^{\Delta}(\lambda) \Delta(\lambda) \\
& =\frac{1}{2} \eta\left(\frac{\varsigma_{1}+\varsigma_{2}}{2}\right)-\frac{1}{\varsigma_{2}-\varsigma_{1}} \int_{\zeta_{1}}^{\frac{\varsigma_{1}+\varsigma_{2}}{2}} \eta^{\diamond}(\lambda) \Delta \lambda+\frac{1}{2} \eta\left(\frac{\varsigma_{1}+\varsigma_{2}}{2}\right)-\frac{1}{\varsigma_{2}-\varsigma_{1}} \int_{\frac{\varsigma_{1}+\varsigma_{2}}{2}}^{\varsigma_{2}} \eta^{\diamond}(\lambda) \Delta \lambda \\
& =\eta\left(\frac{\varsigma_{1}+\varsigma_{2}}{2}\right)-\frac{1}{\varsigma_{2}-\varsigma_{1}} \int_{\zeta_{1}}^{\varsigma_{2}} \eta^{\diamond}(\lambda) \Delta \lambda,
\end{aligned}
$$

which is the required result. 
Corollary 8. Letting $\mathcal{T}=\mathcal{R}$ and making change of variable on right hand side of Lemma 4 , then we attain the following result as a Lemma 2.1 in [35].

$$
\begin{aligned}
& \eta\left(\frac{\varsigma_{1}+\varsigma_{2}}{2}\right)-\frac{1}{\varsigma_{2}-\varsigma_{1}} \int_{\varsigma_{1}}^{\varsigma_{2}} \eta^{\diamond}(\lambda) \Delta \lambda \\
& \leq\left(\varsigma_{2}-\varsigma_{1}\right)\left[\int_{0}^{\frac{1}{2}} r \eta^{\Delta}\left(r \varsigma_{2}+(1-r) \varsigma_{1}\right)+\int_{\frac{1}{2}}^{1}(r-1) \eta^{\Delta}\left(r \varsigma_{2}+(1-r) \varsigma_{1}\right)\right] .
\end{aligned}
$$

Corollary 9. Let $\mathcal{T}=\mathcal{N}$ in Lemma 4 and assume that $\varsigma_{1}=0, \varsigma_{2}=\delta \lambda=\varepsilon$ and $\eta(\kappa)=\lambda_{\kappa}$, then

$$
\lambda_{\frac{\delta}{2}}-\frac{1}{\delta} \sum_{\varepsilon=0}^{\delta} \lambda_{\varepsilon}=\frac{1}{\delta} \sum_{\varepsilon=0}^{\frac{\delta}{2}-1} \varepsilon \Delta \lambda+\frac{1}{\delta} \sum_{\varepsilon=\frac{\delta}{2}}^{\delta-1}(\varepsilon-\delta) \Delta \lambda
$$

Theorem 5. Consider a time scale $\mathcal{T}$ and $Y=\left[\varsigma_{1}, \varsigma_{2}\right] \mathcal{T} \subseteq \mathcal{T}$ such that $\varsigma_{1}<\varsigma_{2}$ and $\varsigma_{1}, \varsigma_{2} \in \mathcal{T}$. Assume that there is a delta differentiable function $\eta: \mathrm{Y} \rightarrow \mathcal{R}$ on $\mathrm{Y}^{\circ}\left(\mathrm{Y}^{\circ}\right.$ is the interior of $\left.\mathrm{Y}\right)$. If $\left|\eta^{\Delta}\right|$ is $\left(\Psi_{1}, \Psi_{2}\right)$-convex, then

$$
\left|\eta\left(\frac{\varsigma_{1}+\varsigma_{2}}{2}\right)-\frac{1}{\varsigma_{2}-\varsigma_{1}} \int_{\varsigma_{1}}^{\varsigma_{2}} \eta^{\diamond}(\lambda) \Delta \lambda\right| \leq\left(\varsigma_{2}-\varsigma_{1}\right)\left[\Omega^{* *}(r)\left|\eta^{\Delta}\left(\varsigma_{1}\right)\right|+\Lambda^{* *}(r)\left|\eta^{\Delta}\left(\varsigma_{2}\right)\right|\right],
$$

where

$$
\begin{aligned}
& \Omega^{* *}(r)=\int_{0}^{\frac{1}{2}} r \Psi_{1}(r) \Psi_{2}(1-r) \Delta r+\int_{\frac{1}{2}}^{1}(1-r) \Psi_{1}(r) \Psi_{2}(1-r) \Delta r, \\
& \Lambda^{* *}(r)=\int_{0}^{\frac{1}{2}} r \Psi_{2}(r) \Psi_{1}(1-r) \Delta r+\int_{\frac{1}{2}}^{1}(1-r) \Psi_{2}(r) \Psi_{1}(1-r) \Delta r .
\end{aligned}
$$

Proof. Using Corollary 8, property of modulus and $\left(\Psi_{1}, \Psi_{2}\right)$-convexity of $\left|\eta^{\Delta}\right|$, we obtain

$$
\begin{aligned}
& \left|\eta\left(\frac{\varsigma_{1}+\varsigma_{2}}{2}\right)-\frac{1}{\varsigma_{2}-\varsigma_{1}} \int_{\varsigma_{1}}^{\varsigma_{2}} \eta^{\diamond}(\lambda) \Delta \lambda\right| \\
& \leq\left(\varsigma_{2}-\varsigma_{1}\right)\left[\int_{0}^{\frac{1}{2}} r\left|\eta^{\Delta}\left(r \varsigma_{2}+(1-r) \varsigma_{1}\right)\right|+\int_{\frac{1}{2}}^{1}|r-1|\left|\eta^{\Delta}\left(r \varsigma_{2}+(1-r) \varsigma_{1}\right)\right|\right] \\
& \leq\left(\varsigma_{2}-\varsigma_{1}\right)\left[\int_{0}^{\frac{1}{2}} r\left\{\Psi_{1}(r) \Psi_{2}(1-r)\left|\eta^{\Delta}\left(\varsigma_{2}\right)\right|+\Psi_{2}(r) \Psi_{1}(1-r)\left|\eta^{\Delta}\left(\varsigma_{1}\right)\right|\right\} \Delta r\right. \\
& \left.\quad+\int_{\frac{1}{2}}^{1}(1-r)\left\{\Psi_{1}(r) \Psi_{2}(1-r)\left|\eta^{\Delta}\left(\varsigma_{2}\right)\right|+\Psi_{2}(r) \Psi_{1}(1-r)\left|\eta^{\Delta}\left(\varsigma_{1}\right)\right|\right\} \Delta r\right] \\
& \leq\left(\varsigma_{2}-\varsigma_{1}\right)\left[\Omega^{* *}(r)\left|\eta^{\Delta}\left(\varsigma_{1}\right)\right|+\Lambda^{* *}(r)\left|\eta^{\Delta}\left(\varsigma_{2}\right)\right|\right],
\end{aligned}
$$

the required result. 
Remark 6. Letting $\mathcal{T}=\mathcal{R}$, then our delta integral reduces to the usual Riemann integral from calculus. Hence, Theorem 5 becomes

$$
\left|\eta\left(\frac{\varsigma_{1}+\varsigma_{2}}{2}\right)-\frac{1}{\varsigma_{2}-\varsigma_{1}} \int_{\varsigma_{1}}^{\varsigma_{2}} \eta(\lambda) \Delta \lambda\right| \leq\left(\varsigma_{2}-\varsigma_{1}\right)\left[\Omega^{* *}(r)\left|\eta^{\prime}\left(\varsigma_{1}\right)\right|+\Lambda^{* *}(r)\left|\eta^{\prime}\left(\varsigma_{2}\right)\right|\right],
$$

where

$$
\begin{aligned}
& \Omega^{* *}(r)=\int_{0}^{\frac{1}{2}} r \Psi_{1}(r) \Psi_{2}(1-r) d r+\int_{\frac{1}{2}}^{1}(1-r) \Psi_{1}(r) \Psi_{2}(1-r) d r, \\
& \Lambda^{* *}(r)=\int_{0}^{\frac{1}{2}} r \Psi_{2}(r) \Psi_{1}(1-r) d r+\int_{\frac{1}{2}}^{1}(1-r) \Psi_{2}(r) \Psi_{1}(1-r) d r .
\end{aligned}
$$

Remark 7. Letting $\mathcal{T}=\mathcal{R}$ along with $\Psi_{1}(r)=r$, and $\Psi_{2}(r)=1$, then Theorem 5 becomes Theorem 2.2 in [35].

Theorem 6. Consider a time scale $\mathcal{T}$ and $\mathrm{Y}=\left[\varsigma_{1}, \varsigma_{2}\right] \mathcal{T} \subseteq \mathcal{T}$ such that $\varsigma_{1}<\varsigma_{2}$ and $\varsigma_{1}, \varsigma_{2} \in \mathcal{T}$. Assume that there is a delta differentiable function $\eta: \mathrm{Y} \rightarrow \mathcal{R}$ on $\mathrm{Y}^{\circ}\left(\mathrm{Y}^{\circ}\right.$ is the interior of $\left.\mathrm{Y}\right)$. If $\left|\eta^{\Delta}\right|$ is $\left(\Psi_{1}, \Psi_{2}\right)$-convex, for $\alpha>1$ such that $\frac{1}{\alpha}+\frac{1}{\beta}=1$, then

$$
\begin{aligned}
& \left|\eta\left(\frac{\varsigma_{1}+\varsigma_{2}}{2}\right)-\frac{1}{\varsigma_{2}-\varsigma_{1}} \int_{\zeta_{1}}^{\varsigma_{2}} \eta^{\diamond}(\lambda) \Delta \lambda\right| \\
& \leq\left(\varsigma_{2}-\varsigma_{1}\right)\left[\left(\int_{0}^{\frac{1}{2}} r^{\alpha} \Delta r\right)^{\frac{1}{\alpha}}\left(\int_{0}^{\frac{1}{2}}\left\{\Psi_{1}(r) \Psi_{2}(1-r)\left|\eta^{\Delta}\left(\varsigma_{2}\right)\right|^{\beta}+\Psi_{2}(r) \Psi_{1}(1-r)\left|\eta^{\Delta}\left(\varsigma_{1}\right)\right| \beta\right\} \Delta r\right)^{\frac{1}{\beta}}\right. \\
& \left.\quad+\left(\int_{\frac{1}{2}}^{1}|1-r|^{\alpha} \Delta r\right)^{\frac{1}{\alpha}}\left(\int_{\frac{1}{2}}^{1}\left\{\Psi_{1}(r) \Psi_{2}(1-r)\left|\eta^{\Delta}\left(\varsigma_{2}\right)\right|^{\beta}+\Psi_{2}(r) \Psi_{1}(1-r)\left|\eta^{\Delta}\left(\varsigma_{1}\right)\right|^{\beta}\right\} \Delta r\right)^{\frac{1}{\beta}}\right] .
\end{aligned}
$$

Proof. Using Corollary 8, the well-known Höulder inequality and $\left(\Psi_{1}, \Psi_{2}\right)$-convexity of $\left|\eta^{\Delta}\right|$, we have

$$
\begin{aligned}
& \left|\eta\left(\frac{\varsigma_{1}+\varsigma_{2}}{2}\right)-\frac{1}{\varsigma_{2}-\varsigma_{1}} \int_{\zeta_{1}}^{\varsigma_{2}} \eta^{\diamond}(\lambda) \Delta \lambda\right| \\
& \leq\left(\varsigma_{2}-\varsigma_{1}\right)\left[\left|\int_{0}^{\frac{1}{2}} r \eta^{\Delta}\left(r \varsigma_{2}+(1-r) \varsigma_{1}\right) \Delta r\right|+\left|\int_{\frac{1}{2}}^{1}(r-1) \eta^{\Delta}\left(r \varsigma_{2}+(1-r) \varsigma_{1}\right) \Delta r\right|\right] \\
& \leq\left(\varsigma_{2}-\varsigma_{1}\right)\left[\left(\int_{0}^{\frac{1}{2}} r^{\alpha} \Delta r\right)^{\frac{1}{\alpha}}\left(\int_{0}^{\frac{1}{2}}\left|\eta^{\Delta}\left(r \varsigma_{2}+(1-r) \varsigma_{1}\right)\right|^{\beta} \Delta r\right)^{\frac{1}{\beta}}\right. \\
& \left.+\left(\int_{\frac{1}{2}}^{1}|1-r|^{\alpha} \Delta r\right)^{\frac{1}{\alpha}}\left(\int_{\frac{1}{2}}^{1}\left|\eta^{\Delta}\left(r \varsigma_{2}+(1-r) \varsigma_{1}\right)\right|^{\beta} \Delta r\right)^{\frac{1}{\beta}}\right]
\end{aligned}
$$




$$
\begin{aligned}
\leq & \left(\varsigma_{2}-\varsigma_{1}\right)\left[\left(\int_{0}^{\frac{1}{2}} r^{\alpha} \Delta r\right)^{\frac{1}{\alpha}}\left(\int_{0}^{\frac{1}{2}}\left\{\Psi_{1}(r) \Psi_{2}(1-r)\left|\eta^{\Delta}\left(\varsigma_{2}\right)\right|^{\beta}+\Psi_{2}(r) \Psi_{1}(1-r)\left|\eta^{\Delta}\left(\varsigma_{1}\right)\right|^{\beta}\right\} \Delta r\right)^{\frac{1}{\beta}}\right. \\
& \left.+\left(\int_{\frac{1}{2}}^{1}|1-r|^{\alpha} \Delta r\right)^{\frac{1}{\alpha}}\left(\int_{\frac{1}{2}}^{1}\left\{\Psi_{1}(r) \Psi_{2}(1-r)\left|\eta^{\Delta}\left(\varsigma_{2}\right)\right|^{\beta}+\Psi_{2}(r) \Psi_{1}(1-r)\left|\eta^{\Delta}\left(\varsigma_{1}\right)\right|^{\beta}\right\} \Delta r\right)^{\frac{1}{\beta}}\right],
\end{aligned}
$$

which is the required result.

Remark 8. Letting $\mathcal{T}=\mathcal{R}$, then our delta integral reduces to the usual Riemann integral from calculus. Hence, Theorem 6 becomes

$$
\begin{aligned}
& \left|\eta\left(\frac{\varsigma_{1}+\varsigma_{2}}{2}\right)-\frac{1}{\varsigma_{2}-\varsigma_{1}} \int_{\varsigma_{1}}^{\varsigma_{2}} \eta(\lambda) d \lambda\right| \\
& \leq\left(\varsigma_{2}-\varsigma_{1}\right)\left[\left(\int_{0}^{\frac{1}{2}} r^{\alpha} d r\right)^{\frac{1}{\alpha}}\left(\int_{0}^{\frac{1}{2}}\left\{\Psi_{1}(r) \Psi_{2}(1-r)\left|\eta^{\prime}\left(\varsigma_{2}\right)\right|^{\beta}+\Psi_{2}(r) \Psi_{1}(1-r)\left|\eta^{\prime}\left(\varsigma_{1}\right)\right|^{\beta}\right\} d r\right)^{\frac{1}{\beta}}\right. \\
& \left.\quad+\left(\int_{\frac{1}{2}}^{1}|1-r|^{\alpha} d r\right)^{\frac{1}{\alpha}}\left(\int_{\frac{1}{2}}^{1}\left\{\Psi_{1}(r) \Psi_{2}(1-r)\left|\eta^{\prime}\left(\varsigma_{2}\right)\right|^{\beta}+\Psi_{2}(r) \Psi_{1}(1-r)\left|\eta^{\prime}\left(\varsigma_{1}\right)\right|^{\beta}\right\} \Delta r\right)^{\frac{1}{\beta}}\right] .
\end{aligned}
$$

Remark 9. Letting $\mathcal{T}=\mathcal{R}$ along with $\Psi_{1}(r)=r$ and $\Psi_{2}(r)=1$, then Theorem 6 becomes Theorem 2.3 in [35].

For our coming result on an arbitrary time scale, we shall use Lemma 1.

Theorem 7. Let $\eta: \mathcal{T} \rightarrow \mathcal{R}$ be a differentiable mapping and $\varsigma_{1}<\varsigma_{2} \in \mathcal{T}$. Let $\left|\eta^{\Delta}\right|$ be $\left(\Psi_{1}, \Psi_{2}\right)$-convexity, then

$$
\begin{aligned}
& \left|\eta\left(\varsigma_{1}\right)\left\{1-\chi_{2}(1,0)\right\}+\eta\left(\varsigma_{2}\right) \chi_{2}(1,0)-\frac{1}{\varsigma_{2}-\varsigma_{1}} \int_{\varsigma_{1}}^{\varsigma_{2}} \eta^{\diamond}(\lambda) \Delta \lambda\right| \\
& \leq \frac{\varsigma_{2}-\varsigma_{1}}{2}\left[\Omega^{* * *}(r, \omega)\left|\eta^{\Delta}\left(\varsigma_{1}\right)\right|+\Lambda^{* * *}(r, \omega)\left|\eta^{\Delta}\left(\varsigma_{2}\right)\right|\right],
\end{aligned}
$$

where

$$
\begin{aligned}
& \Omega^{* * *}(r, \omega)=\int_{0}^{1} \int_{0}^{1}\left\{\Psi_{1}(r) \Psi_{2}(1-r)+\Psi_{1}(\omega) \Psi_{2}(1-\omega)\right\}(r+\omega) \Delta r \Delta \omega, \\
& \Lambda^{* * *}(r, \omega)=\int_{0}^{1} \int_{0}^{1}\left\{\Psi_{2}(r) \Psi_{1}(1-r)+\Psi_{2}(\omega) \Psi_{1}(1-\omega)\right\}(r+\omega) \Delta r \Delta \omega .
\end{aligned}
$$


Proof. Using Lemma 1, modulus property and $\left(\Psi_{1}, \Psi_{2}\right)$-convexity of $\left|\eta^{\Delta}\right|$, we have

$$
\begin{aligned}
\mid & \eta\left(\varsigma_{1}\right)\left\{1-\chi_{2}(1,0)\right\}+\eta\left(\varsigma_{2}\right) \chi_{2}(1,0)-\frac{1}{\varsigma_{2}-\varsigma_{1}} \int_{\varsigma_{1}}^{\varsigma_{2}} \eta^{\diamond}(\lambda) \Delta \lambda \mid \\
\leq & \frac{\varsigma_{2}-\varsigma_{1}}{2} \int_{0}^{1} \int_{0}^{1}\left|\eta^{\Delta}\left(r \varsigma_{1}+(1-r) \varsigma_{2}\right)-\eta^{\Delta}\left(\omega \varsigma_{1}+(1-\omega) \varsigma_{2}\right)\right||\omega-r| \Delta r \Delta \omega \\
\leq & \frac{\varsigma_{2}-\varsigma_{1}}{2} \int_{0}^{1} \int_{0}^{1}\left\{\left|\eta^{\Delta}\left(r \varsigma_{1}+(1-r) \varsigma_{2}\right)+\eta^{\Delta}\left(\omega \varsigma_{1}+(1-\omega) \varsigma_{2}\right)\right|\right\}(r+\omega) \Delta r \Delta \omega \\
\leq & \frac{\varsigma_{2}-\varsigma_{1}}{2} \int_{0}^{1} \int_{0}^{1}\left[\left\{\Psi_{1}(r) \Psi_{2}(1-r)\left|\eta^{\Delta}\left(\varsigma_{1}\right)\right|+\Psi_{2}(r) \Psi_{1}(1-r)\left|\eta^{\Delta}\left(\varsigma_{2}\right)\right|\right\}\right. \\
& \left.+\left\{\Psi_{1}(\omega) \Psi_{2}(1-\omega)\left|\eta^{\Delta}\left(\varsigma_{1}\right)\right|+\Psi_{2}(\omega) \Psi_{1}(1-\omega)\left|\eta^{\Delta}\left(\varsigma_{2}\right)\right|\right\}(r+\omega) \Delta r \Delta \omega\right] \\
\leq & \frac{\varsigma_{2}-\varsigma_{1}}{2} \int_{0}^{1} \int_{0}^{1}\left[\left\{\Psi_{1}(r) \Psi_{2}(1-r)+\Psi_{1}(\omega) \Psi_{2}(1-\omega)\right\}(r+\omega)\left|\eta^{\Delta}\left(\varsigma_{1}\right)\right|\right. \\
& \left.+\left\{\Psi_{2}(r) \Psi_{1}(1-r)+\Psi_{2}(\omega) \Psi_{1}(1-\omega)\right\}(r+\omega)\left|\eta^{\Delta}\left(\varsigma_{2}\right)\right|\right] \Delta r \Delta \omega \\
\leq & \frac{\varsigma_{2}-\varsigma_{1}}{2}\left[\Omega^{* * *}(r, \omega)\left|\eta^{\Delta}\left(\varsigma_{1}\right)\right|+\Lambda^{* * *}(r, \omega)\left|\eta^{\Delta}\left(\varsigma_{2}\right)\right|\right],
\end{aligned}
$$

which is the required result.

Remark 10. Letting $\mathcal{T}=\mathcal{R}$, then our delta integral reduces to the usual Riemann integral from calculus. Hence, Theorem 7 becomes

$$
\left|\frac{\eta\left(\varsigma_{1}\right)+\eta\left(\varsigma_{2}\right)}{2}-\frac{1}{\varsigma_{2}-\varsigma_{1}} \int_{\varsigma_{1}}^{\varsigma_{2}} \eta(\lambda) d \lambda\right| \leq \frac{\varsigma_{2}-\varsigma_{1}}{2}\left[\Omega^{* * *}(r, \omega)\left|\eta^{\prime}\left(\varsigma_{1}\right)\right|+\Lambda^{* * *}(r, \omega)\left|\eta^{\prime}\left(\varsigma_{2}\right)\right|\right],
$$

where

$$
\begin{aligned}
& \Omega^{* * *}(r, \omega)=\int_{0}^{1} \int_{0}^{1}\left\{\Psi_{1}(r) \Psi_{2}(1-r)+\Psi_{1}(\omega) \Psi_{2}(1-\omega)\right\}(r+\omega) d r d \omega, \\
& \Lambda^{* * *}(r, \omega)=\int_{0}^{1} \int_{0}^{1}\left\{\Psi_{2}(r) \Psi_{1}(1-r)+\Psi_{2}(\omega) \Psi_{1}(1-\omega)\right\}(r+\omega) d r d \omega
\end{aligned}
$$

and

$$
\chi_{2}(1,0)=\int_{0}^{1}(1-r) d r=\frac{1}{2}
$$

Remark 11. Letting $\mathcal{T}=\mathcal{R}$ along with $\Psi_{1}(r)=\frac{1}{2}$, and $\Psi_{2}(r)=\frac{1}{4}$, then Theorem 7 becomes Theorem 2.2 in [34].

For our coming result, we shall use Lemma 2. 
Theorem 8. Let $\eta:\left[\varsigma_{1}, \varsigma_{2}\right]_{\mathcal{T}} \subseteq \mathcal{T} \rightarrow \mathcal{R}$ be a delta differentiable mapping on $\mathcal{T}^{\circ}$ and $\varsigma_{1}<\varsigma_{2} \in \mathcal{T}$. Let $\eta^{\Delta} \in \mathbb{C}_{\mathcal{R D}}$, then

$$
\begin{aligned}
& \left|\eta\left(\frac{\varsigma_{1}+\varsigma_{2}}{2}\right)-\frac{1}{\varsigma_{2}-\varsigma_{1}} \int_{\varsigma_{1}}^{\varsigma_{2}} \eta^{\diamond}(\lambda) \Delta \lambda\right| \\
& \leq \frac{\varsigma_{2}-\varsigma_{1}}{2}\left[\Omega^{* * * *}(r, \omega)\left|\eta^{\Delta}\left(\varsigma_{1}\right)\right|+\Lambda^{* * * *}(r, \omega)\left|\eta^{\Delta}\left(\varsigma_{2}\right)\right|\right],
\end{aligned}
$$

where

$$
\begin{aligned}
& \Omega^{* * * *}(r, \omega)=\int_{0}^{1} \int_{0}^{1}(\varphi(\omega)+\varphi(r))\left[\Psi_{1}(r) \Psi_{2}(1-r)+\Psi_{1}(\omega) \Psi_{2}(1-\omega)\right] \Delta r \Delta \omega, \\
& \Lambda^{* * * *}(r, \omega)=\int_{0}^{1} \int_{0}^{1}(\varphi(\omega)+\varphi(r))\left[\Psi_{1}(1-r) \Psi_{2}(r)+\Psi_{1}(1-\omega) \Psi_{2}(\omega)\right] \Delta r \Delta \omega .
\end{aligned}
$$

Proof. Using Lemma 2, modulus property and $\left(\Psi_{1}, \Psi_{2}\right)$-convexity of $\left|\eta^{\Delta}\right|$, we have

$$
\begin{aligned}
\left|\eta\left(\frac{\varsigma_{1}+\varsigma_{2}}{2}\right)-\frac{1}{\varsigma_{2}-\varsigma_{1}} \int_{\varsigma_{1}}^{\varsigma_{2}} \eta^{\diamond}(\lambda) \Delta \lambda\right| \\
\leq \frac{\varsigma_{2}-\varsigma_{1}}{2} \int_{0}^{1} \int_{0}^{1}\left[\left|\eta^{\Delta}\left(r_{1}+(1-r) \varsigma_{2}\right)\right|-\left|\eta^{\Delta}\left(\omega \varsigma_{1}+(1-\omega) \varsigma_{2}\right)\right|\right]|\varphi(\omega)-\varphi(r)| \Delta r \Delta \omega \\
\leq \frac{\varsigma_{2}-\varsigma_{1}}{2} \int_{0}^{1} \int_{0}^{1}\left[\left|\eta^{\Delta}\left(r \varsigma_{1}+(1-r) \varsigma_{2}\right)\right|+\left|\eta^{\Delta}\left(\omega \varsigma_{1}+(1-\omega) \varsigma_{2}\right)\right|\right](\varphi(\omega)-\varphi(r)) \Delta r \Delta \omega \\
\leq \frac{\varsigma_{2}-\varsigma_{1}}{2} \int_{0}^{1} \int_{0}^{1}\left[\left[\Psi_{1}(r) \Psi_{2}(1-r)\left|\eta^{\Delta}\left(\varsigma_{1}\right)\right|+\Psi_{1}(1-r) \Psi_{2}(r)\left|\eta^{\Delta}\left(\varsigma_{2}\right)\right|\right]\right. \\
\left.+\left[\Psi_{1}(\omega) \Psi_{2}(1-\omega)\left|\eta^{\Delta}\left(\varsigma_{1}\right)\right|+\Psi_{1}(1-\omega) \Psi_{2}(\omega)\left|\eta^{\Delta}\left(\varsigma_{2}\right)\right|\right]\right](\varphi(\omega)-\varphi(r)) \Delta r \Delta \omega \\
=\frac{\varsigma_{2}-\varsigma_{1}}{2}\left[\int_{0}^{1} \int_{0}^{1}(\varphi(\omega)-\varphi(r))\left[\Psi_{1}(r) \Psi_{2}(1-r)+\Psi_{1}(\omega) \Psi_{2}(1-\omega)\right]\left|\eta^{\Delta}\left(\varsigma_{1}\right)\right| \Delta r \Delta \omega\right. \\
+\int_{0}^{1} \int_{0}^{1}(\varphi(\omega)-\varphi(r))\left[\Psi_{1}(1-r) \Psi_{2}(r)+\Psi_{1}(1-\omega) \Psi_{2}(\omega)\right]\left|\eta^{\Delta}\left(\varsigma_{2}\right)\right| \Delta r \Delta \omega \\
=\frac{\varsigma_{2}-\varsigma_{1}}{2}\left[\Omega^{* * * *}(r, \omega)\left|\eta^{\Delta}\left(\varsigma_{1}\right)\right|+\Lambda^{* * * *}(r, \omega)\left|\eta^{\Delta}\left(\varsigma_{2}\right)\right|\right],
\end{aligned}
$$

the proof is completed.

Remark 12. Letting $\mathcal{T}=\mathcal{R}$, then our delta integral reduces to the usual Riemann integral from calculus. Hence, Theorem 8 becomes

$$
\begin{aligned}
& \left|\eta\left(\frac{\varsigma_{1}+\varsigma_{2}}{2}\right)-\frac{1}{\varsigma_{2}-\varsigma_{1}} \int_{\varsigma_{1}}^{\varsigma_{2}} \eta(\lambda) \Delta \lambda\right| \\
& \leq \frac{\varsigma_{2}-\varsigma_{1}}{2}\left[\Omega^{* * * *}(r, \omega)\left|\eta^{\prime}\left(\varsigma_{1}\right)\right|+\Lambda^{* * * *}(r, \omega)\left|\eta^{\prime}\left(\varsigma_{2}\right)\right|\right],
\end{aligned}
$$


where

$$
\begin{aligned}
& \Omega^{* * * *}(r, \omega)=\int_{0}^{1} \int_{0}^{1}(\varphi(\omega)-\varphi(r))\left[\Psi_{1}(r) \Psi_{2}(1-r)+\Psi_{1}(\omega) \Psi_{2}(1-\omega)\right] d r d \omega, \\
& \Lambda^{* * * *}(r, \omega)=\int_{0}^{1} \int_{0}^{1}(\varphi(\omega)-\varphi(r))\left[\Psi_{1}(1-r) \Psi_{2}(r)+\Psi_{1}(1-\omega) \Psi_{2}(\omega)\right] d r d \omega .
\end{aligned}
$$

Remark 13. Letting $\mathcal{T}=\mathcal{R}$ along with $\Psi_{1}(r)=\frac{1}{2}$, and $\Psi_{2}(r)=\frac{1}{4}$, then Theorem 8 becomes Theorem 2.2 in [35].

\section{Examples}

Example 2. Let $\mathcal{T}=\mathcal{R}$. Obviously, $\eta(r)=r^{2}$ is convex with $\Psi_{1}(r)=r, \Psi_{2}(r)=1$, and continuous on $(0, \infty)$, so we may apply Theorem 3 with $\varsigma_{1}=0$, and $\varsigma_{2}=2$. Clearly

$$
\begin{aligned}
& \left|\frac{\eta\left(\varsigma_{1}\right)+\eta\left(\varsigma_{2}\right)}{2}-\frac{1}{\varsigma_{2}-\varsigma_{1}} \int_{\varsigma_{1}}^{\varsigma_{2}} \eta \diamond(\lambda) \Delta \lambda\right| \\
& =2-\frac{1}{2} \int_{0}^{2} \lambda^{2} \Delta \lambda \approx 0.6667 .
\end{aligned}
$$

On the other hand

$$
\begin{aligned}
& \frac{\varsigma_{2}-\varsigma_{1}}{2}\left[\Omega^{*}(r)+\Lambda^{*}(r)\right]\left\{\left|\eta^{\Delta}\left(\varsigma_{1}\right)\right|+\left|\eta^{\Delta}\left(\varsigma_{2}\right)\right|\right\} \\
& =\frac{1}{2}[4] \approx 2.0,
\end{aligned}
$$

where

$$
\Omega^{*}(r)=\int_{0}^{1} r^{2} \Delta r, \quad \Lambda^{*}(r)=\int_{0}^{1} r(1-r) \Delta r .
$$

It is nice to see that the following implications hold in (22) and (23),

$$
0.6667<2.0
$$

Example 3. Let $\mathcal{T}=\mathcal{R}$. Obviously, $\eta(r)=\sin r$ is convex with $\Psi_{1}(r)=1, \Psi_{2}(r)=1-r$, and continuous on $(0, \infty)$, so we may apply Theorem 5 with $\varsigma_{1}=0$, and $\varsigma_{2}=\frac{\pi}{6}$. Clearly

$$
\begin{aligned}
& \left|\eta\left(\frac{\varsigma_{1}+\varsigma_{2}}{2}\right)-\frac{1}{\varsigma_{2}-\varsigma_{1}} \int_{\zeta_{1}}^{\varsigma_{2}} \eta\right\rangle(\lambda) \Delta \lambda \mid \\
& =\left|\sin \left(\frac{\pi}{12}\right)+\frac{6}{\pi} \int_{0}^{\frac{\pi}{6}} \sin \lambda \Delta \lambda\right| \approx 0.00294 .
\end{aligned}
$$

On the other hand

$$
\begin{aligned}
& \left(\varsigma_{2}-\varsigma_{1}\right)\left[\Omega^{* *}(r)\left|\eta^{\Delta}\left(\varsigma_{1}\right)\right|+\Lambda^{* *}(r)\left|\eta^{\Delta}\left(\varsigma_{2}\right)\right|\right] \\
& =\frac{\pi}{6}\left[\frac{1}{8}|\cos 0|+\frac{1}{8}\left|\cos \frac{\pi}{6}\right|\right] \approx 0.1221
\end{aligned}
$$


where

$$
\Omega^{* *}(r)=\Lambda^{* *}(r)=\int_{0}^{\frac{1}{2}} r(1-r) \Delta r+\int_{\frac{1}{2}}^{1}(1-r)^{2} \Delta r=\frac{1}{8} .
$$

It is nice to see that the following implications hold in (22) and (23),

$$
0.00294<0.1221 .
$$

Example 4. Let $\mathcal{T}=\mathcal{R}$. Obviously, $\eta(r)=\sqrt{r^{2}+3}$ is convex with $\Psi_{1}(r)=1-r, \Psi_{2}(r)=1$, and continuous on $(0, \infty)$, so we may apply Theorem 7 with $\varsigma_{1}=0.5$, and $\varsigma_{2}=3.5$. Clearly

$$
\begin{aligned}
& \left|\eta\left(\varsigma_{1}\right)\left\{1-\chi_{2}(1,0)\right\}+\eta\left(\varsigma_{2}\right) \chi_{2}(1,0)-\frac{1}{\varsigma_{2}-\varsigma_{1}} \int_{\varsigma_{1}}^{\varsigma_{2}} \eta \diamond(\lambda) \Delta \lambda\right| \\
& =\left|\frac{\sqrt{0.5^{2}+3}}{2}+\frac{\sqrt{3.5^{2}+3}}{2}-\frac{1}{2.5} \int_{0.5}^{3.5} \sqrt{\lambda^{2}+3} \Delta \lambda\right| \\
& \approx 1.2137 .
\end{aligned}
$$

On the other hand

$$
\begin{aligned}
& \frac{\varsigma_{2}-\varsigma_{1}}{2}\left[\Omega^{* * *}(r, \omega)\left|\eta^{\Delta}\left(\varsigma_{1}\right)\right|+\Lambda^{* * *}(r, \omega)\left|\eta^{\Delta}\left(\varsigma_{2}\right)\right|\right] \\
& =\frac{3.5-0.5}{2}\left[\frac{5}{6}\left(\frac{0.5}{\sqrt{0.5^{2}+3}}\right)+\frac{8}{6}\left(\frac{3.5}{\sqrt{3.5^{2}+3}}\right)\right] \\
& \approx 2.1395
\end{aligned}
$$

where

$$
\begin{aligned}
& \Omega^{* * *}(r, \omega)=\int_{0}^{1} \int_{0}^{1}\{(1-r)+(1-\omega)\}(r+\omega) \Delta r \Delta \omega=\frac{5}{6}, \\
& \Lambda^{* * *}(r, \omega)=\int_{0}^{1} \int_{0}^{1}(r+\omega)^{2} \Delta r \Delta \omega=\frac{8}{6} .
\end{aligned}
$$

It is nice to see that the following implications hold in (22) and (23),

$$
1.2137<2.1395 \text {. }
$$




\section{Applications}

We consider the following special means for arbitrary real numbers $\varepsilon, v, \varepsilon \neq v$ :

$$
\begin{aligned}
& \mathcal{A}\left(\xi_{1}, \xi_{2}\right)=\frac{\xi_{1}+\xi_{2}}{2}, \quad \xi_{1}, \xi_{2} \in \mathcal{R}, \\
& \mathcal{G}\left(\xi_{1}, \xi_{2}\right)=\sqrt{\xi_{1} \xi_{2}}, \quad \xi_{1}>0, \xi_{2}>0, \\
& \mathcal{H}\left(\xi_{1}, \xi_{2}\right)=\frac{2 \xi_{1} \xi_{2}}{\xi_{1}+\xi_{2}}, \quad \xi_{1}, \xi_{2} \in \mathcal{R} \backslash\{0\}, \\
& \mathcal{L}\left(\xi_{1}, \xi_{2}\right) \quad=\frac{\xi_{2}-\xi_{1}}{\ln \left|\xi_{2}\right|-\ln \left|\xi_{1}\right|}, \quad\left|\xi_{1}\right| \neq\left|\xi_{2}\right|, \xi_{1} \xi_{2} \neq 0, \\
& \mathcal{L}_{n}\left(\xi_{1}, \xi_{2}\right) \quad=\left[\frac{\xi_{2}^{n+1}-\xi_{1}^{n+1}}{(n+1)\left(\xi_{2}-\xi_{1}\right)}\right], \quad n \in \mathcal{Z} \backslash\{-1,0\}, \xi_{1}, \xi_{2} \in \mathcal{R}, \xi_{1} \neq \xi_{2}, \\
& I\left(\xi_{1}, \xi_{2}\right)=\left\{\begin{array}{cc}
\frac{1}{e}\left(\frac{\xi_{2}^{\xi_{2}}}{\xi_{1}^{\xi_{1}}}\right)^{\frac{1}{\xi_{2}-\xi_{1}}}, & \xi_{1} \neq \xi_{2}, \\
\xi_{1}, & \xi_{1}=\xi_{2} .
\end{array}\right.
\end{aligned}
$$

Presently utilizing outcomes in Section 3, we have a some applications to the special means of real numbers.

Proposition 1. Let $\rho_{1}, \rho_{2} \in \mathcal{R}^{+}, \rho_{1}<\rho_{2}$. then

$$
\left|\mathcal{A}\left(\rho_{1}^{n}, \rho_{2}^{n}\right)-\mathcal{L}_{n}^{n}\left(\rho_{1}, \rho_{2}\right)\right| \leq \frac{n\left(\rho_{2}-\rho_{1}\right)}{12}\left[\left|\rho_{1}\right|^{(n-1)}+\left|\rho_{2}\right|^{(n-1)}\right] .
$$

Proof. Apply Theorem 3 with $\eta(r)=r^{n}, \Psi_{1}(r)=\Psi_{2}(r)=r, \mathcal{T}=\mathcal{R}$ and we get the required result.

Proposition 2. For $\rho_{1}, \rho_{2} \in \mathcal{R}^{+}, \rho_{1}<\rho_{2}$, then

$$
\left|\mathcal{A}\left(e^{\rho_{1}}, e^{\rho_{2}}\right)-\mathcal{L}\left(e^{\rho_{1}}, e^{\rho_{2}}\right)\right| \leq \frac{\rho_{2}-\rho_{1}}{(\alpha+1)^{\frac{1}{\alpha}}}\left[\frac{\left|e^{\rho_{1} \beta}\right|+\left|e^{\rho_{2} \beta}\right|}{6}\right]^{\frac{1}{\beta}} .
$$

Proof. Apply Theorem 4 with $\eta(r)=e^{r}, \Psi_{1}(r)=\Psi_{2}(r)=r, \mathcal{T}=\mathcal{R}$ and we get the required result.

Proposition 3. Let $\rho_{1}, \rho_{2} \in \mathcal{R}^{+}, \rho_{1}<\rho_{2}$. then

$$
\left|\left(\mathcal{A}\left(\rho_{1}, \rho_{2}\right)\right)^{2}-\mathcal{L}_{2}^{2}\left(\rho_{1}, \rho_{2}\right)\right| \leq \frac{5\left(\rho_{2}-\rho_{1}\right)}{48}\left[\left|\rho_{1}\right|+\left|\rho_{2}\right|\right] .
$$

Proof. Apply Theorem 5 with $\eta(r)=r^{2}, \Psi_{1}(r)=\Psi_{2}(r)=r, \mathcal{T}=\mathcal{R}$ and we get the required result.

Proposition 4. For $\rho_{1}, \rho_{2} \in \mathcal{R}^{+}, \rho_{1}<\rho_{2}$, then

$$
\left|\left(\mathcal{A}\left(\rho_{1}, \rho_{2}\right)\right)^{-1}-\mathcal{L}^{-1}\left(\rho_{1}, \rho_{2}\right)\right| \leq \frac{\left(\rho_{2}-\rho_{1}\right)}{(2(\alpha+1))^{\frac{1}{\alpha}}}\left[\frac{\left|\rho_{1}\right|^{2 \beta}+\left|\rho_{2}\right|^{2 \beta}}{12\left(\rho_{1} \rho_{2}\right)^{2 \beta}}\right]^{\frac{1}{\beta}} .
$$

Proof. Apply Theorem 6 with $\eta(r)=\frac{1}{r}, \Psi_{1}(r)=\Psi_{2}(r)=r, \mathcal{T}=\mathcal{R}$ and we get the required result. 
Proposition 5. For $\rho_{1}, \rho_{2} \in \mathcal{R}^{+}, \rho_{1}<\rho_{2}$, then

$$
\left|\mathcal{G}\left(\left(\rho_{1}, \rho_{2}\right)\right)-\ln I\left(\rho_{1}, \rho_{2}\right)\right| \leq \frac{\left(\rho_{2}-\rho_{1}\right)}{4}\left[\frac{\left|\rho_{1}\right|+\left|\rho_{2}\right|}{\left|\rho_{1} \rho_{2}\right|}\right]
$$

Proof. Apply Theorem 7 with $\eta(r)=\ln r, \Psi_{1}(r)=\Psi_{2}(r)=r, \mathcal{T}=\mathcal{R}$ and we get the required result.

Remark 14. Similar technique can be applied to Theorem 8, we get the immediate consequences.

\section{Conclusions}

The principal objective of this article was to derive several variants for $\left(\Psi_{1}, \Psi_{2}\right)$-convex function on time scales. For exceptional appropriate selections of functions $\Psi_{1}$ and $\Psi_{2}$, one can find many novel and existing class of convex functions on time scale as particular cases. This demonstrates that the idea of $\left(\Psi_{1}, \Psi_{2}\right)$-convex function is a broad and modifying one but also improves on some results in the literature on time scale theory. Several particularities were investigated, when $\mathcal{T}=\mathcal{R}$ and $\mathcal{T}=\mathcal{N}$. Lastly, we derived certain dynamic inequalities by utilizing special means.

Author Contributions: All authors contributed substantially to the research. Conceptualization: S.R. and F.S.; methodology, S.R.; software, Y.-M.C. and S.R.; validation, S.R. and M.A.N.; formal analysis, S.R. and M.A.N.; investigation, Y.-M.C., S.R., and M.A.N.; resources, S.R and K.I.N.; data curation, S.R.; writing, original draft preparation, F.S.; writing, review and editing, S.R. and Y.-M.C.; supervision, M.A.N.; project administration, S.R. and K.I.N.; funding acquisition, Y.-M.C.

Funding: This research was funded by the Natural Science Foundation of China grant number 61673169.

Conflicts of Interest: The authors declare no conflict of interest.

\section{References}

1. Hilger, S. Ein Mabkettenkalkul mit Anwendung auf Zentrumsmannigfaltigkeiten. Ph.D. Thesis, Universitot Wurzburg, Wurzburg, Germany, 1988.

2. Bohner, M.; Peterson, A. Dynamic Equation on Time Scale: An Introduction with Application; Birkhauser: Boston, MA, USA, 2001.

3. Bohner, M.; Matthews, T. Ostrowski inequalities on time scales. J. Inequal. Pure Appl. Math. 2008, 9, 8.

4. Dinu, C. Hermite-Hadamard inequality on time scale. J. Inequal. Appl. 2008, 2008, 24. [CrossRef]

5. Dinu, C. Ostrowski type inequalities on time scales. Ann. Univ. Craiova. Math. Comput. Sci. Ser. 2007, 34, 43-58.

6. Bohner, E.A.; Bohner, M.; Akin, F. Pachpatte inequalities on time scales. J. Inequal. Pure Appl. Math. 2005, 6, $1-23$.

7. Donchev, T.; Nosheen, A.; Pecaric, J. Hardy-type inequalities on time scale via convexity in several variables. ISRN Math. Anal. 2013, 9. [CrossRef]

8. Hilger, S. Analysis on measure chains a unified approach to continuous and discrete calculus. Results Math. 1990, 18, 18-56. [CrossRef]

9. Sheng, Q.; Fadag, M.; Henderson, J.; Davis, J.M. An exploration of combined dynamic derivatives on time scales and their applications. Nonlinear Anal. Real World. Appl. 2006, 7, 395-413. [CrossRef]

10. Hilger, S. Differential and difference calculus unified. Nonlinear Anal. 1997, 30, 2683-2694. [CrossRef]

11. Tahir, S.F.; Mushtaq, M.; Muddassar, M. A new interpretation of Hermite-Hadamards type integral inequalities by the way of time scales. J. Comput. Anal. Appl. 2019, 26, 223-233.

12. Agarwal, R.P.; Bohner, M.; Peterson, A. Inequalities on Time Scales: A Survey. Math. Inequal. Appl. 2001, 4, 537-557. [CrossRef]

13. Ammi, M.R.S.; Ferreira, R.A.C.; Torres, D.F.M. Diamond- $\alpha$ Jensens inequality on time scales. J. Inequal. Appl. 2008, 2008, 13. [CrossRef] 
14. Kalsoom, H.; Latif, M.A.; Junjua, M.D.; Hussain, S.; Shahzadi, G. Some $(p, q)$-estimates of Hermite-Hadamard-type inequalities for coordinated convex and quasi-convex functions. Mathematics 2019, 7, 683. [CrossRef]

15. Latif, A.M. On some inequalities for $h$-Convex functions. Int. J. Math. Anal. 2010, 30, 1473-1482.

16. Noor, M.A.; Noor, K.I.; Rashid, S. Some new classes of preinvex functions and inequalities. Mathematics 2019, 7, 29. [CrossRef]

17. Rashid, S.; Noor, M.A.; Noor, K.I. New estimates for exponentially convex functions via conformable fractional operators. Fract. Fract. 2019, 3, 19. [CrossRef]

18. Rashid, S.; Noor, M.A.; Noor, K.I. Some new generalizations for exponentially s-convex functions and inequalities via fractional operators. Fract. Fract. 2019, 3, 24. [CrossRef]

19. Rashid, S.; Noor, M.A.; Noor, K.I. Fractional exponentially $m$-convex functions and inequalities. Int. J. Anal. Appl. 2019, 17, 464-478.

20. Rashid, S.; Akdemir, A.O.; Jarad, F.; Noor, M.A.; Noor, K.I. Simpson's type integral inequalities for $k$-fractional integrals and their applications. AIMS Math. 2019, 4, 1087-1100. [CrossRef]

21. Rashid, S.; Abdeljawad, T.; Jarad, F.; Noor, M.A. Some estimates for generalized Riemann-Liouville fractional integrals of exponentially convex functions and their applications. Mathematics 2019, 7, 807. [CrossRef]

22. Set, E.; Akdemir, A.O.; Mumcu, I. Hadamard's inequality and its extensions for conformable fractional integrals of any order $\alpha>0$. Creat. Math. Inf. 2018, 27, 197-206.

23. Verosanec, S. On h-convexity. J. Math. Anal. Appl. 2007, 326, 303-311. [CrossRef]

24. Hadamard, J. Etude sur les proprietes des fonctions entieres e.t en particulier dune fonction consideree par Riemann. J. Math. Pure Appl. 1893, 58, 171-215.

25. Hermite, C. Sur deux limites dune integrale definie. Mathesis 1883, 3, 82.

26. Alomari, M.W.; Darus, M.; Kirmaci, U.S. Some inequalities of Hermite-Hadamard type for s-convex functions. Acta Math. Sci. 2011, 31B, 1643-1652. [CrossRef]

27. Dragomir, S.S. Some new inequalities of Hermite-Hadamard type for GA-convex functions. Ann. Univ. Mariae Curie Sklodowska 2018, 72, 55-68. [CrossRef]

28. Khan, M.A.; Ali, T.; Khan, T.U. Hermite-Hadamard type inequalities with applications. Fasc. Math. 2017, 59, 57-74. [CrossRef]

29. Niculescu, C.P. The Hermite-Hadamard inequality for convex functions on a global NPC space. J. Math. Anal. Appl. 2009, 356, 295-301. [CrossRef]

30. Dragomir, S.S.; Pearce, C.E.M. Slected Topics on Hermite-Hadamard Inequalities and Applications. In RGMIA Monographs; Victoria University: Footscray, Australia, 2000.

31. Zhang, X.-M.; Chu, Y.-M.; Zhang, X.-H. The Hermite-Hadmard type inequality of GA-convex functions and its application. J. Inequal. Appl. 2010, 2010, 11. [CrossRef]

32. Breckner, W.W. Stetigkeitsaussagen fur eine Klasse verallgemeinerter konvexer funktionen in topologischen linearen Raumen. Publ. Inst. Math. 1978, 23, 13-20.

33. Dragomir, S.S.; Pecaric, J.; Persson, L.E. Some inequalities of Hadamard type. Soochow J. Math. 1995, 21, 335-341.

34. Dragomir, S.S.; Agarwal, R.P. Two inequalities for differentiable mappings and applications to special means of real numbers and to Trapezoidal formula. Appl. Math. Lett. 1998, 11, 91-95. [CrossRef]

35. Kirmaci, U.S. Inequalities for differentiable mappingsand applications to special means of real numbers and to midpoint formula. Appl. Math. Comput. 2004, 47, 137-146.

(c) 2019 by the authors. Licensee MDPI, Basel, Switzerland. This article is an open access article distributed under the terms and conditions of the Creative Commons Attribution (CC BY) license (http://creativecommons.org/licenses/by/4.0/). 